\title{
GESTIÓN EDUCATIVA Y PRÁCTICA DOCENTE: REFLEXIONES SOBRE LA DIMENSIÓN INVESTIGATIVA.
}

\section{EDUCATIONAL MANAGEMENT AND TEACHING PRACTICE: REFLECTIONS ON THE INVESTIGATIVE DIMENSION.}

\section{Autor: Mariela Alexandra Ramírez Zúñiga}

E-mail de contacto: marielaa.ramirez@educacio.es

ORCID ID: https://orcid.org/0000-0002-8661-3742

Articulo recibido: 16 de Mayo del 2019

Articulo revisado: 7 de Julio del 2019

Articulo aprobado: 25 de Diciembre del 2019

Economista egresada de la Universidad de Guayaquil (Ecuador) con 15 años de experiencia en la docencia. Maestrante en la Universidad Nacional Mayor de San Marcos de Lima (Perú), mención Gestión de la Educación.

\section{Resumen}

Los cambios que hay en la sociedad en cuanto a conocimientos y tecnología, demandan de las personas nuevas competencias, por lo cual las instituciones educativas deben responder a ello con una óptima gestión educativa. El docente en particular juega un papel fundamental, ya que es quien tiene el contacto directo con los alumnos y es en el ámbito escolar donde se devela su práctica. A la vista de las consideraciones anteriores, el presente trabajo tiene como propósito reflexionar sobre la inclusión de la dimensión investigativa en la gestión educativa y en la práctica docente. La gestión educativa es uno de los componentes esenciales del sistema educativo, el cual involucra las dimensiones pedagógica-curricular, institucional $\mathrm{u}$ organizativa, administrativa y socialcomunitaria, pero se puede ampliar su campo de acción con la inserción de otra dimensión como es la investigativa, para impulsar la generación de conocimientos y transformaciones consustanciados con la realidad institucional. La práctica docente se expresa en diversas dimensiones: personal, institucional, interpersonal, social, didáctica y valoral; pero lo que podría conducir a la renovación de la práctica docente es un permanente cuestionamiento sobre la misma. Para esto último, se considera relevante la inclusión de la dimensión investigativa en la práctica docente, de modo que los resultados o hallazgos que se obtengan conduzcan al logro de las transformaciones que en materia educativa se requieren. De esta manera, la investigación se presenta como una dimensión que potenciaría una relación sinérgica entre la gestión académica y la práctica docente. Palabras claves: Gestión Educativa; Práctica Docente; Investigación.

Abstract
The changes that exist in society in terms of
knowledge and technology, demand from
people new skills, for which educational
institutions must respond to this with an
optimal educational management. The teacher
in particular plays a fundamental role, since it
is, he who has direct contact with the students,
and it is in the school environment where his
practice is revealed. In view of the above
considerations, this paper aims to reflect on the
inclusion of the research dimension in
educational management and teaching practice.
Educational management is one of the essential
components of the education system, which
involves the pedagogical-curricular,
institutional or organizational, administrative
and social-community dimensions, but its field
of action can be broadened with the insertion of
another dimension such as research. , to
promote the generation of knowledge and
transformations consistent with the
institutional reality. The teaching practice is
expressed in different dimensions: personal,
institutional, interpersonal, social, didactic and
value; but what could lead to the renewal of
teaching practice is a permanent questioning
about it. For the latter, it is considered relevant
the inclusion of the research dimension in
teaching practice, so that the results or findings




\section{Ciencia y Educación (ISSN 2707-3378) \\ Vol. 1 No. 2 \\ Febrero del 2020}

that are obtained, lead to the achievement of transformations that in educational matter are required. In this way, the research is presented as a dimension that would enhance a synergistic relationship between academic management and teaching practice.

\section{Keywords: Educational Management; Teaching Practice; Research.}

\section{Sumário}

As mudanças que existem na sociedade em termos de conhecimento e tecnologia exigem novas habilidades das pessoas, para que as instituições educacionais respondam com ótima gestão educacional. $\mathrm{O}$ professor, em particular, desempenha um papel fundamental, pois é ele quem tem contato direto com os alunos e é no ambiente escolar que sua prática é revelada. Diante do exposto, o presente trabalho tem como objetivo refletir sobre a inclusão da dimensão de pesquisa na gestão educacional e na prática de ensino. A gestão educacional é um dos componentes essenciais do sistema educacional, que envolve as dimensões pedagógico-curricular, institucional ou organizacional, administrativa e socialcomunitária, mas seu campo de ação pode ser ampliado com a inserção de outra dimensão, como a pesquisa, promover a geração de conhecimentos e transformações consolidadas com a realidade institucional. A prática de ensino é expressa em várias dimensões: pessoal, institucional, interpessoal, social, didática e de valor; Mas o que poderia levar à renovação da prática de ensino é um questionamento permanente sobre ela. Para este último, considera-se relevante a inclusão da dimensão de pesquisa na prática docente, de modo que os resultados ou constatações obtidas levem à consecução das transformações necessárias em questões educacionais. Dessa forma, a pesquisa é apresentada como uma dimensão que melhoraria uma relação sinérgica entre gestão acadêmica e prática de ensino.

\section{Palavras-chave: Gestão educacional; Prática de Ensino; Pesquisa.}

\section{Introducción}

A nivel mundial los cambios que hay en la sociedad, los avances en conocimientos y tecnología demandan de las personas nuevas competencias y estar preparadas para afrontar con éxito los retos de la vida. La formación del ser humano está relacionada a la educación que adquiera, es decir, la manera como es educado repercutirá en su realización personal y colectiva. Las instituciones educativas donde los sujetos pasan gran parte de su vida son claves para su desarrollo, por lo tanto, las mismas deben orientarse a desarrollar una óptima gestión educativa.

En concordancia con lo señalado, la gestión educativa se define como a continuación se transcribe:

Proceso sistémico que integra e imprime sentido a las acciones administrativas en el ámbito escolar, con el fin de mejorar las organizaciones, las personas que las integran $\mathrm{y}$ sus propuestas o proyectos educativos, se desarrolla y ejecuta mediante planes, programas y proyectos que optimizan recursos, que generan procesos participativos en beneficio de la comunidad, que interactúan con el medio, que aportan al desarrollo local y regional y que solucionan necesidades educativas en armonía con las necesidades básicas fundamentales del ser humano (Correa et al., 2013, p. 13).

El hecho de que la gestión educativa sea un proceso sistémico implica que no se restringe únicamente a las organizaciones educativas, es decir, no se concibe a éstas en forma aislada, sino que por el contrario, se abordan en función del entorno, de otras organizaciones y en plena interacción con el ámbito local, regional, nacional e incluso mundial. Además, este proceso involucra el desarrollo de planes y proyectos con la participación activa de los actores inmersos en el proceso educativo y en los cuales se administren los recursos 


\section{Ciencia y Educación (ISSN 2707-3378) \\ Vol. 1 No. 2 \\ Febrero del 2020}

efectivamente, de tal modo que se emplee la mínima cantidad de éstos y a la vez se logren los objetivos planteados.

En la gestión que realizan las instituciones educativas confluyen una serie de actores, entre los cuales se encuentran los alumnos, los docentes, los representantes, los directivos y otros actores sociales de la comunidad. Indudablemente, los más importantes son los docentes y los alumnos. El docente en particular juega un papel fundamental, ya que es quien tiene el contacto directo con los alumnos y por tanto, sirve como mediador entre el sistema educativo y éstos. Al respecto, Fierro et al. (1999), citados por Castro (2018) indican el rol que debe cumplir el docente.

El trabajo del maestro está situado en el punto en que se encuentran el sistema escolar (con una oferta curricular y organizativa determinada), y los grupos sociales particulares. En este sentido, su función es mediar el encuentro entre el proyecto político educativo, estructurado como oferta educativa, y sus destinatarios, en una labor que se realiza cara a cara. (p.8)

En función de lo expuesto, la gestión empleada en las instituciones educativas implica las normas y condiciones laborales, la cultura, la interrelación entre los colegas; asimismo, la gestión educativa involucra varias dimensiones, las cuales son: pedagógico-curricular, institucional $\mathrm{u}$ organizativa, administrativa $\mathrm{y}$ social-comunitaria (Alarcón, 2013; Chipana, 2015). La implementación de estas debe orientarse al logro de la eficacia y la eficiencia de las instituciones educativas en particular.

Por otra parte, la práctica docente está asociada a las dimensiones personal, institucional, interpersonal, social, didáctica y valoral (Fierro, 2003). El éxito de la práctica docente depende de qué tan efectiva sea la gestión académica. No obstante, hoy en día las demandas son distintas, pues el vertiginoso avance del conocimiento y la imposibilidad de adaptarse a ello revela la imperiosa necesidad de crear conocimiento, tanto para gestionar las organizaciones como para transformar la práctica docente en consonancia con las demandas de la realidad, y en este sentido, la investigación puede cumplir un papel fundamental. En definitiva, a la vista de las consideraciones anteriores el presente trabajo tiene como objetivo reflexionar sobre la inclusión de la dimensión investigativa en la gestión educativa y en la práctica docente

\section{Gestión educativa}

\section{Desarrollo}

La gestión educativa es de gran importancia para garantizar una educación de calidad en el sistema educativo. Este último posee varios componentes principales e interrelacionados, asimismo requiere de la buena organización y funcionamiento de éstos. Uno de los componentes del sistema educativo es la gestión educativa, la cual se define como "un conjunto de procesos teórico-prácticos integrados $\mathrm{y}$ relacionados, tanto horizontal como verticalmente, dentro del sistema educativo para atender y cumplir las demandas sociales realizadas a la educación" (UNESCO, 1988 citado por Ruiz y Pinchi, 2016, p. 87). En esta definición se destaca lo general en la gestión educativa como un conjunto de procesos, es decir, ésta hace alusión a los grandes fines o demandas que se le hacen al sistema educativo.

En este mismo orden de ideas, Botero (2009) afirma que "la gestión educativa se concibe como el conjunto de procesos, de toma de decisiones y realización de acciones que permiten llevar a cabo las prácticas pedagógicas, su ejecución y evaluación" (p. 2). Esto implica que la gestión educativa es un conjunto de procesos orientados a la ejecución 


\section{Ciencia y Educación (ISSN 2707-3378) \\ Vol. 1 No. 2 \\ Febrero del 2020}

y evaluación de las prácticas pedagógicas, lo cual evidencia una mayor especificidad de ésta.

De manera similar, "la gestión educativa es entendida como un proceso organizado $\mathrm{y}$ orientado a la optimización de procesos y proyectos internos de las instituciones, con el objetivo de perfeccionar los procedimientos pedagógicos, directivos, comunitarios y administrativos que en ella se movilizan" (Rico, 2016, p. 57). En este caso, la definición igualmente concibe la gestión educativa como un proceso que se desarrolla de manera específica en las instituciones y que tiene como propósito elevar la calidad en cuanto a la práctica pedagógica, la gerencia, la relación escuela-comunidad y las funciones administrativas.

Sobre la base de esta perspectiva, la gestión educativa no se ubica en la mera teoría, sino que la trasciende, se vincula a la práctica cotidiana y toma en consideración las políticas o directrices que establece el Estado. La gestión educativa va más allá de las simples funciones administrativas que tradicionalmente se cumplen, como son la planificación, la ejecución, el control y la evaluación; pues aborda también la práctica pedagógica, la relación escuela-comunidad y las funciones directivas, esto sin dejar de lado los grandes fines o propósitos de la educación y las expectativas que la sociedad ha puesto en la educación, de modo tal que logre conjugar la teoría y la práctica.

Sobre la base de las indagaciones realizadas, la gestión educativa se define como un conjunto de procesos administrativos que se dirigen a la gestión institucional, pero también engloba las actividades para coordinar e impulsar los aspectos pedagógicos, comunitarios y de liderazgo que desde una perspectiva teórico- práctica se despliegan en las organizaciones educativas con el fin último de alcanzar los grandes fines de la educación y por ende satisfacer las demandas educativas de la sociedad.

\section{Objetivos de la gestión educativa}

Alcanzar el desarrollo de una gestión educativa adecuada amerita la consecución de un conjunto de objetivos y en este sentido se coincide con las ideas desarrolladas por el Ministerio de Educación (2003) de Perú, instancia que considera relevantes los objetivos que se enfocan en los siguientes aspectos:

$>$ Desarrollar la institución educativa como una comunidad de aprendizaje encargada de lograr una excelente calidad educativa.

$>$ Fortalecer la capacidad de decisión de las instituciones educativas para que actúen con autonomía pedagógica y administrativa. Las funciones de todas las instancias de gestión se rigen por los principios de subsidiariedad, solidaridad, complementariedad y concurrencia.

$>$ Asegurar la coherencia de las disposiciones administrativas y la subordinación de éstas a las decisiones de carácter pedagógico.

$>$ Lograr el manejo eficaz, eficiente e innovador de las instituciones educativas, de modo que conduzca a la excelencia educativa.

$>$ Desarrollar liderazgos democráticos.

$>$ Colaborar en la articulación intersectorial, de manera que se garanticen los procesos de gestión en el marco de una política de desarrollo integral del país.

$>$ Promover la activa participación de la comunidad.

> Articular las instituciones educativas para que desarrollen relaciones de cooperación y solidaridad.

$>$ Fortalecer el ejercicio ético de las funciones administrativas para favorecer la 


\section{Ciencia y Educación (ISSN 2707-3378) \\ Vol. 1 No. 2 \\ Febrero del 2020}

transparencia y el libre acceso a la información.

$>$ Participar en el efectivo funcionamiento de los mecanismos para prevenir y sancionar los actos de corrupción en la gestión.

$>$ Incentivar la autoevaluación y evaluación permanentes que garanticen el logro de las metas y objetivos establecidos por la institución educativa.

\section{Principios de la Gestión Educativa}

La gestión educativa se rige por un conjunto de normas, valores, creencias y supuestos en torno a los cuales se consideran relevantes las argumentaciones de Alarcón (2013), quien se guía por los siguientes principios: estructuras participativas, de comunicación horizontal, que privilegian la creatividad y el compromiso colectivo; organización concebida como un sistema abierto al aprendizaje, gestión centrada en los beneficiarios, mejoramiento continuo como estrategia de cambio permanente, con la finalidad de prestar servicios de calidad, conforme a las demandas cambiantes de los beneficiarios; desarrollo del personal a través de la calificación creciente, ajustada a necesidades de la organización; cooperación-negociación como forma de elevar la efectividad y los beneficios mutuos, apertura a la comunidad, orientaciones en relación a la planificación y gestión caracterizadas por la acción y el conocimiento científico, la necesidad de enfrentar incertidumbres de sistemas complejos, el arte de modelar estrategias en el marco de una gestión flexible y el desarrollo de habilidades gerenciales como la construcción de la visión, la misión, la comunicación, la motivación, la toma de decisiones y la asignación de recursos.

En consonancia con lo señalado, también se coincide con Obregón (2002), quien especifica que la gestión en el proceso educativo se orienta por los siguientes principios:

$>$ Gestión centrada en los estudiantes. La razón de ser de las instituciones educativas son los estudiantes y todas las acciones de su conducción deben ser canalizadas para lograr este objetivo institucional. La organización, las normas, los reglamentos, los procedimientos, el sistema de control y las acciones cotidianas no deben contradecir los objetivos establecidos en los estatutos y leyes correspondientes.

$>$ Jerarquía y autoridad claramente definidas. Permite garantizar la unidad de acción de la organización, en la cual la dirección dirige, impulsa y coordina sin disminuir las competencias de cada instancia.

$>$ Determinación de quién y cómo se toman las decisiones. Esto significa determinar la responsabilidad que le corresponde a cada persona, estamento, comisión y equipo en la toma de decisiones y resultados que se obtengan.

> Claridad en la definición de canales de participación. Para que la participación de los miembros de la comunidad educativa guarde coherencia con los objetivos institucionales, se deben establecer sistemas bien definidos, cada miembro debe conocer las formas, los momentos de su participación y la contribución coherente que debe tener con los objetivos institucionales, saber dónde, cuándo, cómo, porqué participar y qué resultados se esperan obtener.

$>$ Ubicación del personal de acuerdo con su competencia y/o especialización. Se refiere a la necesidad de tener en cuenta las habilidades y competencias de cada persona para considerar su ubicación en el lugar donde pueda tener un mejor rendimiento y realización, lo cual 


\section{Ciencia y Educación (ISSN 2707-3378) \\ Vol. 1 No. 2 \\ Febrero del 2020}

contribuirá a optimizar el funcionamiento de la organización.

Coordinación fluida y bien definida. Establecer instancias de coordinación ágil y oportuna, mejorar la sincronización de acciones, evitar esfuerzos innecesarios y permitir una mejor acción conjunta.

$>$ Transparencia y comunicación permanente. Todas las acciones que se realicen a nivel de la organización educativa deben ser conocidas por los miembros de la comunidad. Esto contribuye a tener un clima favorable de relaciones, evitando sospechas, malentendidos y acusaciones innecesarias.

Control y evaluación eficaces y oportunos. Para un mejoramiento continuo, el control debe proporcionar información que oriente de manera oportuna las decisiones y asegure la dirección que tomen las tareas en función de los objetivos institucionales.

\section{Dimensiones de la gestión educativa}

El desarrollo de la gestión educativa se ha dividido en varias dimensiones para poder discriminar los diferentes aspectos que contempla. En general, las dimensiones de la gestión educativa son la pedagógica o pedagógica curricular, la organizativaadministrativa y la de participación social, comunitaria o social-comunitaria, las cuales se describen a continuación.

Pedagógica-curricular: Se refiere a los quehaceres educativos y sus actores. Se enfoca hacia la gestión del proceso enseñanzaaprendizaje. Guarda relación con las formas o estilos de enseñanza (metodología, técnicas) y aprendizaje de los alumnos (Alarcón, 2013).

La gestión pedagógica impulsa la práctica docente, implica cómo desarrollar el proceso de enseñanza, cómo se concibe el currículo, cómo se plasma este último en la planificación y cómo se evalúa; asimismo, se contempla la interacción docente-alumnos y docente-padres de familia con el propósito de lograr un proceso de aprendizaje óptimo (Gobierno Federal de los Estados Unidos Mexicanos, 2010).

Con relación a esta dimensión, se reconoce que es el proceso fundamental del quehacer de la institución educativa y los miembros que la integran. Ésta involucra el proceso enseñanzaaprendizaje, la diversificación curricular, las estrategias metodológicas y didácticas, la evaluación del aprendizaje, el uso de materiales educativos y recursos didácticos. Asimismo, comprende la labor del docente, las prácticas pedagógicas, el uso de planes y programas, el manejo de enfoques pedagógicos y estrategias didácticas, los estilos de enseñanza, las relaciones con los estudiantes, la formación y actualización docente para fortalecer sus competencias. Entre las actividades que se contemplan en esta dimensión se encuentran las siguientes: orientación educativa y tutoría, enfoque de evaluación, actualización docente, estilo de enseñanza, relación con los estudiantes, enfoque pedagógico, estrategias didácticas y procesos de enseñanza-aprendizaje, planes y programas (Chipana, 2015).

Organizativa-administrativa: Es el aspecto de las organizaciones que considera las funciones de planeación, organización, dirección y monitoreo como parte medular de las instituciones. Además de asegurar acciones de mejora y cumplimiento de normas y políticas (Alarcón, 2013).

La gestión de las instituciones educativas comprende acciones de orden administrativo, gerencial, de política de personal, económicopresupuestarias, de planificación, de programación, de regulación y de orientación, 


\section{Ciencia y Educación (ISSN 2707-3378) \\ Vol. 1 No. 2 \\ Febrero del 2020}

entre otras (Gobierno Federal de los Estados Unidos Mexicanos, 2010).

Por otra parte, Chipana (2015) hace referencia a una dimensión institucional y a una dimensión administrativa. La dimensión institucional ofrece un marco para la sistematización y el análisis de las acciones referidas a aquellos aspectos de estructura de un centro educativo, entre los cuales se tienen los que pertenecen a la estructura formal (los organigramas, la distribución de tareas y la división del trabajo, el uso del tiempo y de los espacios) y los que conforman la estructura informal (vínculos, formas de relacionarse, y estilos en las prácticas cotidianas, ritos y ceremonias que identifican a la institución). Específicamente, abarca el uso del tiempo y los espacios, los canales de comunicación formal, las comisiones de trabajo, el manual de funciones $\mathrm{y}$ procedimientos, el reglamento interno y los organigramas.

Adicionalmente, se converge con la postura de Chipana (2015), para quien la dimensión administrativa incluye acciones y estrategias de conducción de los recursos humanos, materiales y económicos; procesos técnicos, de tiempo, de seguridad e higiene y control de la información relacionada a todos los miembros de la institución educativa; de igual manera el cumplimiento de la normatividad y la supervisión de las funciones con el único objetivo de favorecer los procesos de enseñanza-aprendizaje. Algunas de las funciones que se realizan son la administración del personal, el mantenimiento y conservación de los bienes muebles e inmuebles, organización de la información y aspectos documentarios de la institución; elaboración de presupuestos y todo el manejo contablefinanciero. Particularmente, se contemplan aspectos como: presupuesto económico, distribución de tiempos, jornadas de trabajo, servicios administrativos, administración de recursos materiales, relación con instancias del Ministerio de Educación u organismo análogo.

Participación social-comunitaria: Es una de las funciones que tiene la institución educativa para con la comunidad, se encarga de velar por la eficaz atención a los usuarios, la convivencia de los usuarios del servicio (alumnos) y la inclusión, tal y como lo expresa Alarcón (2013).

La gestión escolar o socio-comunitaria alude al conjunto de acciones que realizan los actores del hecho educativo con el propósito de generar las condiciones, ambientes y procesos requeridos para que se desarrollen los aprendizajes conforme a los fines, propósitos y objetivos de la educación (Gobierno Federal de los Estados Unidos Mexicanos, 2010).

Asimismo, se converge con los planteamientos de Chipana (2015), para quien la dimensión comunitaria hace referencia a la forma en la que la institución se relaciona con la comunidad conociendo y comprendiendo sus condiciones, necesidades y demandas; asimismo, cómo se integra y participa de la cultura comunitaria. De igual forma se refiere a las relaciones de la institución educativa con el entorno social e interinstitucional, considerando a los padres de familia y organizaciones de la comunidad, municipales, estatales, organizacionales civiles y eclesiales, entre otras. La participación de los mismos debe responder a un objetivo que facilite entablar alianzas estratégicas para el mejoramiento de la calidad educativa. Entre las actividades que se realizan en esta dimensión, se encuentran las siguientes: relación de padres y madres de familia, proyectos de proyección social, redes con instituciones municipales, estatales, eclesiásticas y organismos civiles. 


\section{Modelos de gestión educativa}

Una de las dificultades que generalmente se ha presentado en la gestión educativa es que se ha tratado de desarrollar a la luz de modelos extrapolados desde las ciencias administrativas, y la adhesión a éstos ha generado la desvinculación con la realidad educativa y la absurda aplicación de recetas exógenas e inapropiadas.

De este modo, se presentan entre diversos modelos de gestión educativa, los más representativos: el normativo o tradicional y el estratégico o estratégico-participativo (Gobierno Federal de los Estados Unidos Mexicanos, 2010), los cuales pueden vincularse a los paradigmas positivista y postpositivista, respectivamente.

Coincidiendo con Martínez (2002), la aplicación del paradigma científico-positivista resaltó una visión mecanicista que, condujo al desarrollo industrial, al avance vertiginoso de la ciencia, la tecnología y la innovación, a la prevalencia de la democracia liberal y al individualismo, pero ya no brinda respuestas adecuadas a la realidad actual, sus patrones se han resquebrajado y las perturbaciones son impresionantes: guerras, xenofobia, contaminación, racismo, confrontaciones políticas y religiosas, pobreza, hambre, nacionalismo exacerbado y desarrollo nuclear y armamentista con riesgo de destrucción planetaria, entre otros aspectos.

Por otra parte, el paradigma del postpositivismo se inserta en la posmodernidad, conforme a la cual se trata de representar la realidad como integradora, estructural, sistémica, con una óptica de valoración de los seres vivos y de las cosas, considerando los eventos y las personas por lo que son en sí, hace énfasis en la red de relaciones, utiliza estrategias para comprender la inmensa y compleja red de relaciones en torno a las realidades y propende a las transformaciones y cambios en la sociedad.

Desde las argumentaciones realizadas, el modelo normativo para la gestión educativa estaría caracterizado por las relaciones jerárquicas entre los actores, la prevalencia de un liderazgo autocrático por parte del rector y los otros directivos, la adecuación a las normativas legales, la gestión de un currículo fragmentado en disciplinas como estancos aislados, la resistencia al cambio, la comunicación de tipo vertical con escasa o nula participación del docente y otros actores, la concepción de una gestión pedagógica en la cual los alumnos son considerados como recipientes donde los docentes depositan sus conocimientos para que sean memorizados y repetidos por los primeros, la inflexibilidad para probar estrategias didácticas o modelos pedagógicos distintos y la desvinculación con la comunidad, entre otros aspectos.

En contraposición con el modelo anterior, está el estratégico-participativo, el cual propende a la relación dialógica entre los actores, la aplicación de liderazgos democráticos o transformacionales que instan al resto de los actores a convertirse también en líderes, sin obviar las normativas legales; se tiende al logro de la misión, visión, objetivos institucionales a través de proyectos estratégicos y acciones en forma consensuada; se fomentan acciones para aplicar estrategias o principios curriculares como la transversalidad, núcleos problemáticos o proyectos que conduzcan a la recuperación de la visión de totalidad del conocimiento y por ende a la superación de la visión fragmentada del currículo por parte de los docentes; el 


\section{Ciencia y Educación (ISSN 2707-3378) \\ Vol. 1 No. 2 \\ Febrero del 2020}

manejo del cambio como oportunidad para innovar, la aplicación de un estilo de comunicación horizontal que fluya por toda la organización y con otras organizaciones, la concepción de una gestión pedagógica donde los alumnos se convierten en los actores fundamentales para la construcción del conocimiento desde sus experiencias y del significado que tenga para ellos, la apertura para adaptar y crear nuevas estrategias didácticas o modelos pedagógicos, la vinculación con la comunidad para la resolución de problemas y otros aspectos que permitan impulsar el diálogo, la discusión, la participación, el consenso y la emancipación.

La dimensión investigativa en la gestión educativa para trascender a un modelo de gestión más participativo

Dado que los cambios a nivel global se producen a una velocidad tal que las organizaciones no se pueden adaptar a éstos, lo relevante sería poder crear conocimiento a partir de la realidad concreta de las instituciones educativas y lograr una mayor calidad en su funcionamiento. En función de esto y en concordancia con las ideas desarrolladas, vale la pena destacar que para lograr un proceso de gestión educativa cónsono con las transformaciones tan rápidas que están ocurriendo en la sociedad y con el propósito de fomentar la participación consensuada de los actores, no son suficientes las dimensiones que fueron descritas previamente; es menester incluir una nueva dimensión que no solo sea una parcela más, sino que permee e integre todas las dimensiones expuestas por los autores citados, como lo es la investigativa.

La función de investigación es propia del nivel de educación superior o universitaria. De allí que "la gestión educativa en este ámbito contribuye a la optimización de procesos académicos, pedagógicos, administrativos y financieros" (Rico, 2016, p. 62). Pero, esto generalmente no ocurre así en los otros niveles del sistema educativo correspondiente a los países de Latinoamérica. No obstante, es la gestión de la investigación la que puede -en interacción con las dimensiones restantescontribuir en la gestión educativa para la resolución de las problemáticas que afectan la realidad en este ámbito.

Más allá de los objetivos que comúnmente se le atribuyen a la gestión educativa, el propósito de impulsar procesos de investigación como otra dimensión de la gestión educativa, se convierte en una prioridad si realmente se desea el cambio en los diferentes niveles del sistema educativo y no únicamente en el universitario. Para lograr este cometido, será menester promover procesos de actualización docente orientados al desarrollo de competencias investigativas en los directivos y docentes, fomentando la investigación no solo como práctica de gestión, sino ir más allá y lograr su utilización como práctica pedagógica. Si la investigación permea toda la organización educativa junto a los actores comunitarios, esto redundaría en la formación de un gran talento humano en todo el ámbito educativo, tanto al interior de las organizaciones como fuera de éstas.

Lo señalado anteriormente, permite destacar que la gestión educativa no solamente está dirigida a la ampliación o acondicionamiento de la infraestructura o a la adquisición de recursos materiales, sino que va más allá, de modo que la formación del talento humano se convierta en la prioridad de la organización, y que por tanto, las funciones administrativas inherentes a la planificación, organización, dirección, ejecución y evaluación deben estar enfocadas al desarrollo de diversas competencias en los 


\section{Ciencia y Educación (ISSN 2707-3378) \\ Vol. 1 No. 2 \\ Febrero del 2020}

actores, entre las cuales se destaca la investigativa.

A diferencia de otro tipo de organizaciones, en las educativas, la razón de ser son los seres humanos. Por tanto, la gestión que se emprenda debe partir de su realidad, contemplar la participación de los diferentes actores y consustanciarse con los grandes fines $\mathrm{y}$ propósitos de la educación. Es indudable que la investigación brindaría un aporte fundamental para alcanzar este cometido, por cuanto todos los actores (alumnos, directivos, docentes, administrativos, obreros y otros actores de la comunidad) desempeñarán un rol protagónico para develar su realidad y en función de ello, podrán emprender acciones para transformarla. En este sentido, cobra vital importancia la actualización de los docentes con respecto a lo que implica la investigación, sus bondades y las repercusiones positivas que pudiese tener en la gestión educativa. La idea central es gestionar las instituciones desde y para la investigación.

Como una tarea pendiente para desarrollar y consolidar la gestión educativa en el contexto latinoamericano, se considera que:

Los investigadores en gestión educativa que viven y trabajan en América Latina debieran desarrollar programas de investigación y una base de conocimientos que sean compatibles con lo que se respeta y considera efectivo en la cultura y la tradición latinoamericanas. También, debieran rechazar las ideologías foráneas y las soluciones importadas para los problemas en gestión educativa y brindar los fundamentos para una base de conocimientos en gestión educativa enraizada en las narrativas históricas locales y en los contextos sociales. (Oplatka, 2019, p. 206)

Lo descrito, implica que la investigación debe ser contextualizada y ejecutada por uno o más actores de las instituciones educativas, pero el rol protagónico lo lleva el docente como investigador, es decir, ésta se convierte en otra función a cumplir y que debe ser impulsada por los directivos, a la vez ayuda a elevar la calidad de las otras dimensiones, pero también mejora la investigación como dimensión, esto último implica saber cómo se desarrolla la investigación en las instituciones educativas.

La inclusión de la dimensión investigativa en la gestión educativa permitiría satisfacer los requerimientos de eficacia, eficiencia, productividad, rentabilidad, demandas sociales y empresariales, entre otros aspectos que se vinculan al sector educativo. El propósito es tratar de que las organizaciones educativas se deslastren de las meras recetas que son impuestas inclusive desde instancias gubernamentales y se propenda al cambio, pero desde y para la realidad en la cual se encuentran inmersas las instituciones. A través de esta dimensión, las instituciones tienen la posibilidad de gestionar recursos, así como diseñar, proponer y promover estrategias para monitorear de forma científica lo que ocurre en las otras dimensiones de la gestión educativa, de tal modo que se elaboren diagnósticos, se desarrollen y evalúen propuestas curriculares, se detecte cómo funcionan los procesos administrativos y gerenciales, se hagan proyecciones estadísticas sobre ingreso, prosecución y egreso estudiantil, se emprendan procesos de transformación en cualquier ámbito de la educación.

Aunado a lo planteado, otro de los beneficios de la inclusión de la dimensión investigativa es el relativo al surgimiento e implementación de procesos de innovación que inclusive pueden ser emulados por otras organizaciones educativas y por tanto, contribuir al logro de una educación de calidad. 


\section{Ciencia y Educación (ISSN 2707-3378) \\ Vol. 1 No. 2 \\ Febrero del 2020}

Sobre la base de lo referido, se han seleccionado las dimensiones pedagógica-curricular, institucional $\mathrm{u}$ organizativa, administrativa, social-comunitaria e investigativa como aquellas que desde la perspectiva de la autora se consideran apropiadas para desarrollar la gestión educativa. En la Figura 1 se representan gráficamente tales dimensiones.

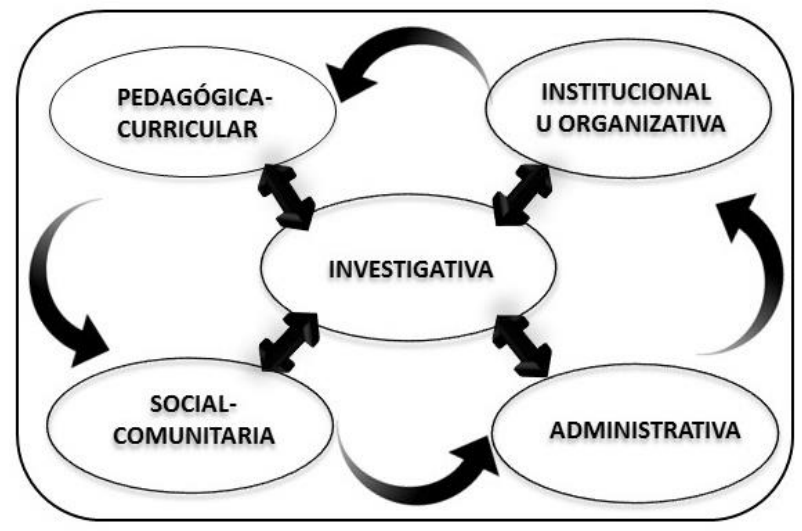

Figura 1. Dimensiones de la gestión educativa

Fuente: La autora con aportes de Alarcón (2013), Gobierno Federal de los Estados Unidos Mexicanos (2010) y Chipana (2015).

Específicamente, la investigación es un proceso sistemático que tiene como propósito generar conocimiento original, formular enunciados o postulados, aportar teorías y solucionar problemas desde la perspectiva del método científico. Las investigaciones se pueden realizar desde la óptica de diversos paradigmas, tanto positivistas (empírico-inductivo y racionalista deductivo) como postpositivistas (fenomenológico vivencial, reflexivo crítico y holístico o complejo) que demandan determinadas metodologías, técnicas e instrumentos. Además, según el nivel de profundización en el conocimiento, se concuerda con Hurtado (2010), quien refiere varios tipos de investigación que se pueden llevar a cabo, las cuales son: exploratoria, descriptiva, analítica, comparativa, explicativa, explicativa, predictiva, proyectiva, interactiva, confirmatoria y evaluativa.

El nivel exploratorio se aplica cuando la temática de investigación no ha sido estudiada o se ha estudiado muy poco. La investigación descriptiva alude a las características de un fenómeno objeto de estudio. La analítica pretende descomponer la realidad en sus elementos constituyentes y establecer relaciones en función de ciertas categorías de análisis. La comparativa persigue la búsqueda de similitudes y discrepancias entre grupos investigados. La explicativa se enfoca en la producción o generación de teorías. La predictiva tiene como propósito establecer escenarios futuros para determinadas situaciones en estudio. La proyectiva se realiza con la finalidad de generar proyectos, propuestas o diseños orientados a la resolución de situaciones problemáticas. En la interactiva, el responsable del estudio junto a los actores de una comunidad u organización interactúan para generar transformaciones en torno a una situación problemática. La confirmatoria es la típica investigación enmarcada en el positivismo y fue la única aceptada durante mucho tiempo. En este tipo de investigación se someten a la refutación las teorías. La evaluativa se desarrolla después de haber puesto en marcha o ejecución un plan, programa, proyecto o diseño; con el fin de constatar su efectividad.

Los diferentes niveles de investigación se pueden aplicar en todas las dimensiones de la gestión educativa y fortalecer la práctica docente. En el cuadro 1 se detallan algunos ejemplos de posibles investigaciones que se pueden desarrollar en el marco de cada una de las dimensiones de la gestión educativa descritas anteriormente y su correspondiente nivel de investigación. 


\section{Ciencia y Educación (ISSN 2707-3378) \\ Vol. 1 No. 2 \\ Febrero del 2020}

\section{Práctica docente}

Si bien, la gestión educativa busca impulsar una serie de acciones y estrategias dirigidas a la obtención y administración de recursos, tanto humanos como materiales de forma eficiente, así como a la formación del talento humano y la implementación del currículo; es en la práctica docente donde se materializa o expresa la calidad de esa gestión educativa.

La práctica docente es más que un simple hacer de los docentes. Para comprender su importancia es necesario articular hechos educativos con el contexto en el cual se producen y los significados que adquieren en el mismo, es decir, que en todo proceso educativo existe un entrelazamiento de contextos: criterios institucionales, organizativos, tradiciones metodológicas, interpretaciones de los profesores, condiciones ambientales y curriculares. Asimismo, existen actividades conscientes e intencionales que admiten esquemas teóricos previos explícitos o implícitos, esos esquemas no se obtienen de forma aislada, sino que se aprenden y se comparten con otros profesionales.

La práctica docente es de carácter social, objetivo e intencional. En ella intervienen los significados, percepciones y acciones de las personas involucradas en el proceso educativo (alumnos, docentes, padres, autoridades, entre otros). También intervienen los aspectos político-institucionales, administrativos y normativos, que en virtud del proyecto educativo de cada centro delimitan el rol del profesor. Es decir, la práctica docente supone una diversa gama de relaciones entre personas. (Muñoz et al., 2016, p. 80)

Reflexionar sobre lo que es la práctica docente implica aceptar que ésta es dinámica, que tiene que ver con el docente y con todos los actores del hecho educativo. Adicionalmente, involucra no solamente el sentir del docente, su paradigma y estilo; sino que también se ve impregnada por las políticas, los fines de las instituciones, así como todos los preceptos normativos y administrativos correspondientes, aunado a los factores económicos, políticos, sociales, culturales e históricos en los cuales se encuentra inmersa la sociedad y que por tanto demandan del docente su participación para satisfacer las necesidades que se generan en estos contextos, pero que también afectan su labor pedagógica.

Las acciones de enseñanza en la práctica docente son muchas y de diversa índole: las acciones lógicas de enseñar, informar, explicar, describir, ejemplificar y mostrar, entre otras; y aquellas acciones de naturaleza más gerencial como: controlar, motivar y evaluar. Por otra parte, vista la práctica como un proceso de resolución de problemas, el profesor queda en un papel de agente, capaz de tomar decisiones y resolver dificultades (Gómez y Polanía, 2008). Del mismo modo, el docente se convierte en un ejemplo, un modelo a seguir por los valores que practica y promueve.

En este sentido, la acción educativa no es un mero hacer sino que supone historia y tradición. En la práctica educativa los esquemas de pensamiento aprendidos son problemáticos, en la medida en que están sujetos a procesos reflexivos y deliberativos, abiertos a interpretaciones diversas y divergentes en virtud de las construcciones individuales y grupales de las comunidades en las que se articulan. Por lo anterior se puede señalar que la práctica docente por su conformación es heterogénea e histórica, y refleja los significados de los cuales se han apropiado los profesores durante su vida profesional. En el proceso de apropiación, los maestros se 


\section{Ciencia y Educación (ISSN 2707-3378) \\ Vol. 1 No. 2 \\ Febrero del 2020}

confrontan con los significados del oficio, rechazan algunos, integran otros a su propia práctica y generan a su vez nuevos significados al enfrentarse a la resolución de múltiples asuntos en su rol educativo (Vergara, 2005).

Desde una perspectiva crítica y reflexiva, la práctica docente propende a la emancipación del ser humano, tal y como lo refieren Castro et al. (2006).

La práctica docente se entiende como una acción que permite innovar, profundizar y transformar el proceso de enseñanza del docente en el aula, asimismo la práctica docente está unida a la realidad del aula, debido a que todo lo que hace el docente se refiere a lo que se hace en la vida cotidiana de la escuela, esta inscripción hace posible una producción de conocimientos a partir del abordaje de la práctica docente como un objeto de conocimiento para los sujetos que intervienen, por eso la práctica se debe delimitar en el orden de la praxis como proceso de comprensión, creación y transformación de un aspecto de la realidad educativa. La práctica del docente se considera como la emancipación profesional para elaborar crítica, reflexiva $y$ eficazmente un estilo de enseñanza que promueva un aprendizaje significativo en los alumnos y logre un pensamiento-acción innovador, trabajando en equipo para desarrollar un proyecto educativo común. (p. 584)

Esto significa que el docente no está anclado a un modelo epistémico de carácter positivista conforme al cual solo sería un transmisor de conocimientos y se comportaría como un líder de carácter autocrático. Por el contrario, el docente en su práctica debe propender a un estilo más humanista, comprometido con la transformación de la sociedad y actuando con una perspectiva crítica y reflexiva, pero también liberadora, que esté consustanciado con las demandas de los nuevos tiempos.

Página 60
A la vista de las consideraciones anteriores, la práctica docente no es rígida, la misma es inseparable de la reflexión que sobre ésta se hace y los procesos de transformación que se emprendan. Y es que el docente es el primero que debe permanentemente estar cuestionando su práctica, pues eso es lo que le permitirá adaptarse a los cambios e impulsar nuevas ideas.

Son diversos los actores involucrados en la práctica docente y al respecto, Fierro (2003) señala que los docentes establecen vínculos con otras personas para alcanzar los objetivos, competencias y fines educativos previstos. Esta interacción se produce con los alumnos y alumnas, otros maestros, padres de familia, autoridades educativas, administradores educativos, miembros de la comunidad y cualquier otro tipo de personal adscrito a la institución. La escuela se configura como el escenario de formación continua del maestro y recrea el marco normativo y la regulación administrativa del sistema.

\section{La inclusión de la dimensión investigativa en la práctica docente}

Es importante resaltar que las reflexiones sobre la práctica docente deben ser sistematizadas, de forma tal que los resultados o hallazgos a los cuales se llegue sirvan para fortalecerla. Desde la práctica se pueden generar nuevos conocimientos e innovaciones educativas que conduzcan a la tan ansiada calidad educativa, pero para ello se considera necesario incluir la dimensión investigativa, donde el educador se ubicaría en el ámbito de acción correspondiente al de docente-investigador. Desde la gestión educativa se impulsaría la investigación y en la práctica docente se materializaría la ejecución de proyectos de investigación y la productividad de los mismos (presentaciones, 


\section{Ciencia y Educación (ISSN 2707-3378) \\ Vol. 1 No. 2 \\ Febrero del 2020}

resúmenes y extensos para memorias de eventos de eventos científicos, artículos científicos, nuevas propuestas pedagógicas, informes sobre diagnósticos o descripciones de las distintas funciones administrativas que se implementan en las instituciones educativas, propuestas y evaluaciones curriculares, entre otras).

Algunos pudieran pensar que la investigación no se puede desarrollar junto al resto de las actividades que debe cumplir el docente, o que esta función deben cumplirla los investigadores de esta área del conocimiento, a pesar de estar fuera de la realidad particular en la cual se desenvuelven los educadores. Sin embargo, desde la perspectiva que se maneja en el presente artículo, se considera que no deben ser investigadores ajenos a los establecimientos educativos quienes deben abordar los procesos de investigación. Por el contrario, esta tarea le corresponde a los propios docentes y directivos en conjunto con el resto de los actores pertenecientes a las comunidades educativas, de manera que ayuden al fortalecimiento de la práctica y a la generación de modelos y teorías que bien pudiesen ser aplicados en otros espacios institucionales, pero adaptándolos a la realidad.

La inclusión de la dimensión investigativa facilitaría la generación de conocimiento original y novedoso, cónsono con el contexto institucional, local, regional, nacional y latinoamericano. Al respecto, y a pesar de que no se considera la investigación como una dimensión de la práctica docente en la mayoría de los establecimientos educativos, se reconoce que "la investigación latinoamericana en gestión educativa debe asumir una mayor responsabilidad y compromiso con el desempeño escolar y los temas de política de América Latina" (Oplatka, 2019, p. 205).
Sobre la base de las apreciaciones realizadas, se considera fundamental la asunción de la dimensión investigativa en la práctica docente, de tal forma que se hagan estudios directamente con los actores involucrados en la realidad educativa que se considere. Adicionalmente, es importante recalcar que la investigación puede realizarse sobre todas las dimensiones de la gestión educativa y de la práctica docente descrita e inclusive sobre la investigación como dimensión de ésta, porque también sería menester establecer las necesidades, requerimientos, debilidades, fortalezas y éxitos en los procesos de investigación.

En este sentido, la gestión educativa debe apalancar los procesos de investigación contribuyendo con la formación en investigación del personal, si éste no la tiene, así como también facilitando las oportunidades, recursos, estrategias y demás requerimientos para que los docentes puedan fortalecer e innovar en su práctica, de tal forma que esto redunde en la calidad de la educación que se brinde.

La investigación se presenta así como una dimensión que se nutre de las otras dimensiones expuestas por los autores, pero que de manera simultánea las integra y le brinda insumos para promover transformaciones, o que a través de ésta, se logren los cambios, como por ejemplo, bajo la modalidad de investigación-acción, con la cual a la par que se investiga también se transforma la realidad. Todo depende del paradigma y el tipo de investigación que se implemente. Cabe destacar que en educación debe tenerse suficiente apertura para aplicar cualquier modalidad de investigación que permita alcanzar los objetivos de la investigación. 


\section{Ciencia y Educación (ISSN 2707-3378) \\ Vol. 1 No. 2 \\ Febrero del 2020}

Es así como se devela que la gestión educativa y la práctica docente impregnadas por la investigación como una dimensión en ambas, pero con propósitos distintos que se complementan (en la primera se gestiona y en la segunda se ejecuta), constituye una alternativa que bien pudiera ser aplicada en las instituciones de todos los niveles del sistema educativo, no solamente en el nivel superior o universitario.

\section{Conclusión}

La gestión educativa es uno de los componentes esenciales del sistema educativo, el cual involucra las dimensiones pedagógicacurricular, institucional $\mathrm{u}$ organizativa, administrativa y social-comunitaria, pero se puede ampliar su campo de acción con la inserción de otra dimensión como lo es la investigativa, desde la cual se pueden diseñar estrategias, sugerir necesidades y facilitar la formación del talento humano, así como gestionar los recursos materiales para impulsar procesos de investigación que redunden en la generación de conocimientos y transformaciones consustanciados con la realidad institucional.

La práctica docente se expresa en diversas dimensiones: personal, institucional, interpersonal, social, didáctica y valoral; pero lo que podría conducir a la renovación de la práctica docente es un permanente cuestionamiento sobre la misma, el cual debe trascender de una mera reflexión a un proceso sistematizado sobre lo que sucede con respecto a la misma y a los actores involucrados en el hecho educativo. Indudablemente, para alcanzar el propósito señalado es necesario acudir a la investigación, de allí que se considere relevante la inclusión de la dimensión investigativa en la práctica docente. De este modo, desde la investigación se puede lograr la concreción de una nueva práctica pedagógica que se adapte a los nuevos tiempos o que se proyecte hacia nuevos escenarios mediante la utilización de conocimiento novedoso y develado en el propio contexto institucional, esto con el fin de lograr las transformaciones que en materia educativa se requieran.

Los resultados o hallazgos que se obtengan a través de procesos de investigación en las organizaciones educativas constituyen los insumos necesarios para transformar no solamente la práctica docente a través de nuevas estrategias, prácticas pedagógicas, modelos curriculares u otros; sino que también pueden aportar información valiosa en torno a los niveles de inclusión, prosecución o repitencia, entre otros; y con respecto a las funciones administrativas de planificación, organización, ejecución, evaluación, liderazgo, toma de decisiones, gestión financiera $\mathrm{u}$ otro componente del proceso de gestión educativa. De manera que a partir de los resultados que aporta la investigación se pueda mejorar la gestión educativa, pues los propósitos $\mathrm{u}$ objetivos estarán suficientemente sustentados e imbricados en la realidad institucional. De este modo, la investigación se presenta como una dimensión que potenciaría una relación sinérgica entre la gestión académica y la práctica docente.

\section{Referencias bibliográficas}

Alarcón, S. (2013). Gestión Educativa y Calidad de la Educación en instituciones privadas en Lima Metropolitana (Tesis de Maestría). Universidad de San Martín de Porres, Lima. Recuperado de http://www.repositorioacademico.usmp.edu. pe/bitstream/usmp/1168/1/alarcon_msz.pdf

Botero, C. (2009). Cinco tendencias de la gestión educativa. Revista Iberoamericana de Educación. 49(2), 1-11. Recuperado de: https://rieoei.org/RIE/article/view/2100 


\section{Ciencia y Educación (ISSN 2707-3378) \\ Vol. 1 No. 2 \\ Febrero del 2020}

Castro, R. (2018). El desarrollo de habilidades docentes y asesoría académica del alumnado de escuelas normales públicas mexicanas en colegios de Murcia España: una experiencia exitosa. RIDE. Revista Iberoamericana para la Investigación y el Desarrollo Educativo, 8(16), 394-410. Recuperado de: http://www.ride.org.mx/index.php/RIDE/art icle/view/347/1623

Castro, E., Peley, R. y Morillo, R. (2006). La práctica pedagógica y el desarrollo de estrategias instruccionales desde el enfoque constructivista. Revista de Ciencias Sociales, 12(3), 581-587. Recuperado de: http://www.scielo.org.ve/scielo.php?script= sci_arttext\&pid=S1315-

95182006000300012

Chipana, M. (2015). Gestión Pedagógica y la Calidad Educativa en las Unidades de Gestión Educativa de San Román y Azángaro - 2013. (Tesis doctoral), Universidad Andina "Néstor Cáceres Velásquez", Juliaca. Recuperado de: http://repositorio.uancv.edu.pe/handle/UAN CV/276

Correa, A., Álvarez, A. y Correa, S. (2013). La gestión educativa un nuevo paradigma. Medellín, Colombia: Fundación Universitaria Luis Amigó. Recuperado de: http://virtual.funlam.edu.co/repositorio/sites /default/files/6lagestioneducativaunnuevopa radigma.pdf

Fierro, J. (2003). La práctica docente y sus dimensiones. Valoras, Pontificia Universidad Católica de Chile. Recuperado de:

http://www.academia.edu/25350876/La_pr \%C3\%A1ctica_docente_y_sus_dimensione $\mathrm{s}$

Gobierno Federal de México (2009). El Modelo de Gestión Educativa Estratégica. Ciudad de México: Secretaría de Educación Pública. Recuperado de: http://formacion.sigeyucatan.gob.mx/forma cion/materiales/5/d3/p3/3.\%20EL\%20MOD ELO\%20DE\%20GESTION\%20EDUCATI VA\%20ESTRATEGICA.pdf

Gómez, M. y Polanía, N. (2008). Estilos de enseñanza y modelos pedagógicos: un estudio con profesores del Programa de
Ingeniería Financiera de la Universidad Piloto de Colombia (Tesis de Maestría). Universidad de La Salle, Bogotá. Recuperado de: http://repository.lasalle.edu.co/handle/1018 $5 / 1667$

Hurtado, J. (2010). Metodología de la investigación. Caracas, Venezuela: Quirón Ediciones.

Martínez, M. (2002). La nueva ciencia. Su desafío, lógica y método. México: Trillas.

Ministerio de Educación (2003). Ley General de Educación. Ley 28044. Lima, Perú: Ministerio de Educación. Recuperado de: http://www.minedu.gob.pe/p/ley_general_d e_educacion_28044.pdf

Muñoz Olivero, J., Villagra Bravo, C. y Sepúlveda Silva, S. (2016). Proceso de reflexión docente para mejorar las prácticas de evaluación de aprendizaje en el contexto de la educación para jóvenes y adultos (EPJA). Folios, (44), 77-91. Recuperado de: http://www.scielo.org.co/scielo.php?script= sci_arttext\&pid=S0123-

$48702016000200005 \& \operatorname{lng}=\mathrm{en} \& \operatorname{tlng}=\mathrm{es}$.

Obregón, N. (2002). Influencia del currículo y del sistema de soporte en la calidad de la gestión administrativa en la Facultad de Educación de la UNFV (Tesis de Maestría). Universidad Nacional Mayor de San Marcos, Perú. Recuperado de: http://cybertesis.unmsm.edu.pe/bitstream/ha ndle/cybertesis/1676/Obregon_an.pdf?seque nce $=1 \&$ is Allowed $=y$

Oplatka, I. (2019). El surgimiento de la gestión educativa como campo de estudio en América Latina. Revista Eletrônica de Educação, 13(1), 196-210. Recuperado de: http://www.reveduc.ufscar.br/index.php/rev educ/article/view/3072

Rico, A. (2018). La gestión educativa: Hacia la optimización de la formación docente en la educación superior en Colombia. Sophia, 12(1), 55-70. Recuperado de: http://www.scielo.org.co/pdf/sph/v12n1/v12 n1a04.pdf

Ruiz, C. y Pinchi, W. (2016). Impacto de la cultura organizacional en la gestión educativa de la Unidad de Gestión Educativa Local de Ascope - 2014. Ciencia y 
Tecnología, 12(1), 85-99. Recuperado de: http://revistas.unitru.edu.pe/index.php/PGM /article/viewFile/1362/1394

Vergara, M. (2005). Significados de la práctica docente que tienen los profesores de educación primaria REICE. Revista Electrónica Iberoamericana sobre Calidad, Eficacia y Cambio en Educación, 3(1), 685-
697.

Recuperado

de:

http://www.ice.deusto.es/rinace/reice/vol3n 1_e/Vergara.pdf

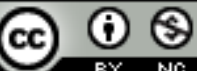

Esta obra está bajo una licencia de Creative Commons Reconocimiento-No Comercial 4.0 Internacional. Copyright (c) Mariela Alexandra Ramírez Zúñiga 
